\section{mCRC: KRAS-Mutationen und Erhaltungstherapie}

\begin{abstract}
Kann man bei Patienten mit einem mCRC nach Induktionstherapie die am besten geeignete Erhaltungstherapie anhand des KRAS-Mutationsstatus ermitteln? In der Nordic ACT2-Studie wurde das untersucht.
\end{abstract}

$\mathrm{E}^{\mathrm{c}}$ ckpfeiler in der Versorgung nicht mehr kurativ behandelbarer Patienten mit metastasiertem Kolorektalkarzinom (mCRC) ist die kombinierte Chemotherapie mit oder ohne zielgerichtete Substanzen. Große klinische Studien zu kombinierten Erhaltungstherapien gibt es jedoch nicht, auch ist die Bedeutung von Biomarkern für den Einsatz gezielter Substanzen in der Erhaltung nicht gut verstanden.

Mutationen in RAS-Genen erwiesen sich z.B. als negative prädiktive Faktoren für EGFR(„epidermal growth factor receptor")-Antikörper beim mCRC, aber als positive Prädiktoren für EGFRTyrosinkinasehemmer (TKI) wie Gefitinib oder Erlotinib bei Patienten mit nichtkleinzelligem Lungenkarzinom. In der 2010 initiierten Nordic ACT2-Studie wurde der Nutzen einer Erlotinib-Addition zu dem gegen VEGF („vascular endothelial growth factor") gerichteten Antikörper Bevacizumab in der Erhaltungstherapie bei Patienten mit KRASWildtyp(Wt)-Tumoren untersucht. Die 233 mCRC-Patienten hatten eine 18-wöchige Induktionstherapie mit XELOX/ FOLFOX oder XELIRI/FOLFIRI plus Bevacizumab absolviert und darunter keinen Progress gezeigt. Patienten mit KRAS-Wt bekamen eine Erhaltungstherapie mit Bevacizumab alleine oder Bevacizumab plus Erlotinib, Patienten mit KRAS-mutiertem (mut) Tumor Bevacizumab alleine oder kontinuierlich Capecitabin alleine. Primärer Endpunkt war das progressionsfreie Überleben (PFS) 3 Monate nach Beginn der Erhaltung.
Bei Patienten mit KRAS-Wt brachte zusätzliches Erlotinib in der Erhaltung im Vergleich zu Bevacizumab allein keine Vorteile bei PFS oder Gesamtüberleben, steigerte aber die Toxizität. $\mathrm{Ob}$ metronomisches Capecitabin bei Patienten mit KRASmut-Tumoren das Ergebnis gegenüber Bevacizumab verbessert, konnte aufgrund der explorativen Statistik nicht geklärt werden. Der Ansatz sollte aber wegen der guten Verträglichkeit von Capecitabin weiter verfolgt werden.

Fazit: Der KRAS-Mutationsstatus beeinflusst das Ergebnis einer ErlotinibAddition in der Erhaltungstherapie bei mCRC-Patienten nicht entscheidend. Capecitabin ist bei Patienten mit Mutation womöglich nicht weniger wirksam als Bevacizumab, aber besser verträglich.

Barbara Kreutzkamp

Hagman $\mathrm{H}$ et al. A randomized study of KRAS-guided maintenance therapy with bevacizumab, erlotinib or metronomic capecitabine after first-line induction treatment of metastatic colorectal cancer: the Nordic ACT2 trial. Ann Oncol. 2016;27(1):140-7.

\section{EGFR-Liganden-Expression bei mCRC assoziiert mit dem Ansprechen im CT}

\begin{abstract}
Können EGFR-Liganden wie EREG bei Patienten mit metastasiertem kolorektalem Karzinom als prognostische Marker auch für eine Erstlinien-Chemotherapie herangezogen werden? Eine Analyse der FIRE 1-Studie suggeriert „ja“.
\end{abstract}

\footnotetext{
A mphiregulin (AREG) und EpireA gulin (EREG) sind 2 Liganden des EGF(„epidermal growth factor“)-Rezeptors, die bisher vor allem als Prädiktoren für eine Cetuximab-Behandlung bei $\mathrm{Pa}$ tienten mit metastasiertem Kolorektalkarzinom (mCRC) untersucht wurden. Bei verstärkter Expression der Liganden ist mit einem besseren Ergebnis unter der Antikörpertherapie zu rechnen. Ob AREG und EREG auch prognostische Bedeutung für die Patienten haben, ist bislang jedoch unklar. Auf Basis der Daten von FIRE 1, einer multizentrischen Studie, sollte deshalb der Zusammenhang zwischen AREG/EREG-Expression und dem Therapieergebnis von mCRC-Patienten unter einer Erstlinien-
}

Chemotherapie mit 5-Fluorouracil/Folinsäure plus Irinotecan (FUFIRI) oder Irinotecan plus Oxaliplatin (mIROX) analysiert werden. In molekularen Untersuchungen an Tumoren von 208 Studienteilnehmern ermittelten die Forscher neben der mRNA-Expression von EREG und AREG zusätzlich die Expression von EGFR sowie Mutationen u.a. der RAS-Gene. Sie korrelierten die Ergebnisse mit den klinischen Resultaten nach FUFIRI bzw. mIROX hinsichtlich der Faktoren Ansprechen, progressionsfreies (PFS) und Gesamtüberleben (OS).

Eine hohe AREG-Expression war mit einem signifikant besseren PFS assoziiert (10,0 vs. 8,0 Monate; Hazard Ratio [HR] $0,62 ; \mathrm{p}=0,03)$. Das OS betrug 24,6 ver- sus 18,7 Monate (HR 0,72; $\mathrm{p}=0,11$ ). Auch eine hohe EREG-Expression korrelierte mit einem längeren PFS (9,4 vs. 6,8 Monate; HR 0,$62 ; \mathrm{p}=0,002$ ) sowie hier auch signifikant mit einem längeren $\mathrm{OS}(25,8$, vs. 15,5 Monate; HR 0,48; $\mathrm{p}<0,001)$. Der positive prognostische Effekt einer hohen EREG-Expression bei der Chemotherapie des mCRC bestätigte sich in multivariaten Analysen und interferierte nicht mit der EGFR-Expression oder $\mathrm{Mu}$ tationen von RAS- und PIK3CA-Genen.

Fazit: Die Expression des EGFR-Liganden Epiregulin (EREG) scheint ein unabhängiger positiver prognostischer Marker für den Erfolg einer Irinotecanbasierten Erstlinien-Chemotherapie beim mCRC zu sein. Die EGFR-Expression beeinflusst diesen Zusammenhang nicht.

Barbara Kreutzkamp

Stahler A et al. Influence of mRNA expression of epiregulin and amphiregulin on outcome of patients with metastatic colorectal cancer treated with 5-FU/LV plus irinotecan or irinotecan plus oxaliplatin as first-line treatment (FIRE 1-trial). Int J Cancer. 2016;138(3):739-46. 